\title{
HYDRAULIQUE
}

\section{Le dessableur, les turbines et la production d'énergie de l'Usine de Marlengo}

\author{
par Henri Dufour, Ingénieur S. I. A. à Lausanne
}

\section{INTRODUCTION}

Les usines hydro-électriques de $T e l(H=70 \mathrm{~m}$.) et, immédiatement en aval, de Marlengo $(H=130 \mathrm{~m}$.), sur la haute Adige, utilisent un débil de $35 \mathrm{~m}^{3} / \mathrm{sec}$. ol produluisent ensemble une puissance de $80.000 \mathrm{ch}$. Après l'agrandissement de la première el la construction de la seconde vers 1925 , ces deux usines disposaient d'une prise d'eau commune représentée sur les fig. 1 et 2.

Pour supprimer l'usure, par endroils très marquée, des galeries, des canaux, des conduites forcées et atténurer celle des turbines, excessivement rapide et onéreuse, dont souffraient les deux usines, la "Societì Alto Adige " du Groupe "Montecalini "à Milan, propriélaire de l'Usine de Marlengo, a fail conslyuire de 1929 à 1931 le dessableur de Tel selon les plans et avec les pièces spéciales que nous lui avons livrés. Elle a bien voulu nous autoriser à publier les résultats qui ront suivre et nous nous faisons un plaisir de, lui en exprimer ici nos remerciements bien sincères.

$\mathrm{Vu}$ le manque de place entre la prise d'eau et l'entrée du tunnel, le dessableur a dù être construit en souterrain dans l'éperon de rocher visible sur la fig. 1. Pour ne pas interrompre l'exploitation des usines, il fallut exécuter d'abord le bassin $B q$ avec sa vanne d'entrée $V_{1}$, sa galerie d'amenée, sa vanne de sortie $V_{2}$, son raccordement à la galerie de dérivation vers l'usine et sa galerie d'évacuation. L'cau ayant été détournée par le bassin $B g$ terminé, on put ensuite, sous la protection de sa vanne d'entrée $V_{1}$ et d'un batardeau $b$, exécuter le bassin $B d$. L'exploitation de ce grand dessableur à purge automatique et continue, excavé entièrement dans le gneiss et dont la fig. 3 donne une vue intérieure, ayant donné toute satisfaction, il nous a paru intéressant d'en faire connaitre l'efficacité et les résultats pratiques très importants pour l'Usine de Marlengo. 


\section{EFFICAGITÉ DU DESSABLEUR}

Dans le but de vérifier la garantie d'efficacité donnée par l'inventeur, la "Società Alto Adige " fit procéder, le $1^{\text {er }}$ juillet 1931, en présence de ses représentants, d'une délégation des "Azienda Elettrica Consorziale " des villes de Bolzano et

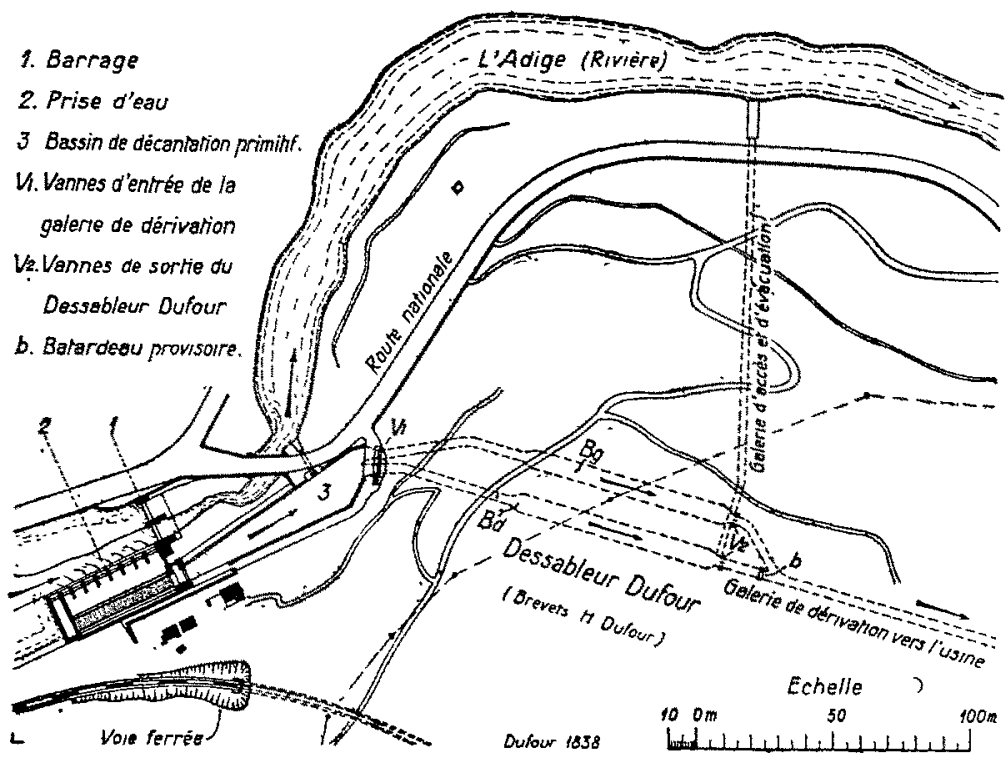

Fig. 1. - Plan de situation de la prise d'eau et du dessableur de Tel (sur l'Adige) pour $35 \mathrm{~m}^{3} / \mathrm{sec}$.

de Merano, propriétaires de I'Usine de Tel, et de deux délégués du "Corpo Reale del Genio-Civile " de la province de Trento, à des épreuves de réception du dessableur. Par suite de la fonte des neiges et des glaciers, l'Adige était, ce jour-là, très grosse ; les usines étaient en marche à pleine charge, le dessableur débitait environ $37 \mathrm{~m}^{3} / \mathrm{sec}$.

Les épreuves consistaient à prélever, dans l'axe vertical de la section de sortie d'un des bassins du dessableur, un peu en amont de la vanne $V_{2}$, au moyen d'un dispositif spécial, au fond, au milieu et à la surface de l'eau, à intervalles réguliers, pendant trois heures, un volume total d'eau dessablée de 1.500 litres. Au moyen d'un autre dispositif situé à la sortie de la galerie d'évacuation, il fut prélevé, simultanément et pendant le même temps, un volume total d'eau do purge de 1.400 litres. (Cette inégalité des volumes d'eau prélevés résulte de la différence de capacité des récipients utilisés.)

Le volume du résidu de 1.500 litres d'eau dessablée a été de $119,95 \mathrm{~cm}^{3}$, sa composition granulométrique la suivante :

\section{Tableau 1}

Diamètre des grains

$$
\begin{aligned}
& 0-0,1 \mathrm{~mm} . \\
& 0,1-0,2 " \\
& 0,2-0,35 " \\
& 0,35-0,5 " \\
& >0,5 \mathrm{~mm} .
\end{aligned}
$$

Volumes partiels

$$
\begin{array}{r}
81,55 \% \\
15,16 ” \\
2,54 ” \\
0,67 ” \\
0,08 \%
\end{array}
$$

$100,00 \%$

Le résidu des 1.400 litres d'eau de purge contenait des grains de loutes les dimensions entre ceux du limon le plus fin et ceux du gravier dont la plus grosse pierre mesurait $50 \times 30 \times 15 \mathrm{~mm}$. Multipliés, pour la comparaison avec le résidu de l'eau dessablée, par le rapport $1,5: 1,4$, les résultats de l'analyse du résidu de l'eau de purge ont été les suivants :

Sur un volume tolal de résidu de $2.518 \mathrm{~cm}^{3}, 1.714,5 \mathrm{~cm}^{3}$, soit le $68 \%$, passaient par le tamis à mailles carrées de $0,5 \mathrm{~mm}$., le reste, soit $803,5 \mathrm{~cm}^{3}$, ou le $32 \%$ était plus grossier.

La composition granulomélrique des $1.714,5 \mathrm{~cm}^{3}$ de résidu dont les grains étaient $<0,5 \mathrm{~mm}$., a été la suivante :

\section{TABLEAU 2}

Diamètre des grains

$$
\begin{aligned}
& 0-0,1 \mathrm{~mm} . \\
& 0,1-0,2 " \\
& 0,2-0,35 " \\
& 0,35-0,5 "
\end{aligned}
$$

\begin{tabular}{|c|c|c|}
\hline $0-0,1 \mathrm{~mm}$ & $97,80 \mathrm{~cm}^{3}$ & $203,5 \mathrm{~cm}^{3}$ \\
\hline $0,1-0,2 "$ & $18,20 \quad "$ & $422,0 \quad »$ \\
\hline $0,2-0,35 n$ & $3,05 \quad n$ & 512,0 \\
\hline $0,35-0,5 \quad$ & 0,80 & $577,0 \quad$ \\
\hline & $119,85 \mathrm{~cm}^{3}$ & $14,5 \mathrm{~cm}^{3}$ \\
\hline
\end{tabular}

Volumes partiels

$$
\begin{aligned}
& 11,89 \% \\
& 24,60 " \\
& 29,84 \% \\
& 33,67 "
\end{aligned}
$$$$
100,00 \%
$$

Le tableau 3 donne, pour leur comparaison, les volumes des diverses catégories de grains $<0,5 \mathrm{~mm}$. contenus dans 1.500 litres d'eau dessablée et dans 1.500 litres d'eau de purge.

\section{Tableau 3}

Volumes des résidus contenus dans 1.5001 . d'eau dessablée d'eau de purge

Diamètre des gnains

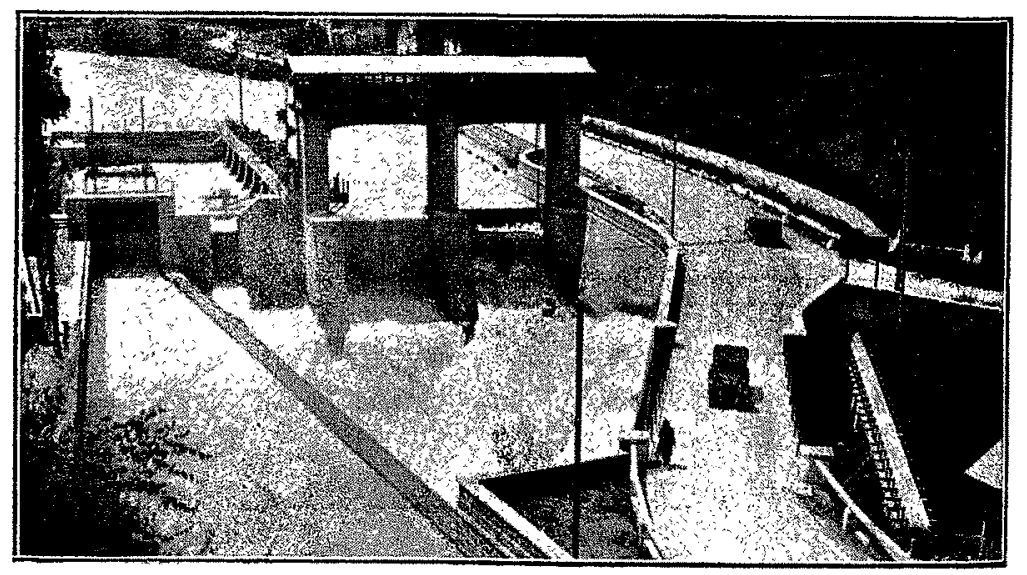

Fig. 2. - Prise d'eau de Tel (sur l'Adige) pour $35 \mathrm{~m}^{3} / \mathrm{sec}$. Barrage à deux vannes, vannes d'entrée, grille horizontale, vanne d'admission, bassin de décantation primitif.

Le tableau 3 montre que 1.500 litres d'eau de purge contenaient $203,5 \mathrm{~cm}^{3}$ de grains $<0,1 \mathrm{~mm}$. alors que $1.500 \mathrm{li}-$ tres d'eau dessablée n'en contenaient que $97,8 \mathrm{~cm}^{3}$. Le même phénomène, mais toujours plus fortement caractérisé, sq retrouve dans les catégories de grains de 0,1 à $0,2 \mathrm{~mm}$., 0,2 à $0,35 \mathrm{~mm}$. et 0,35 à $0,5 \mathrm{~mm}$. Dé ces chiffres, il résulte 
que le dessubleur ćlimine non sculement tous les grains dépassant $0,5 \mathrm{~mm}$., mais aussi une très forte proportion de grains cntre 0,5 et $0,35 \mathrm{~mm}$., 0,35 et $0,2 \mathrm{~mm}$., etc..., et même, encore une forte quantité de grains compris entre 0,1 et $0 \mathrm{~mm}$.

De celte efficacité très poussée du dessableur, il résulte que

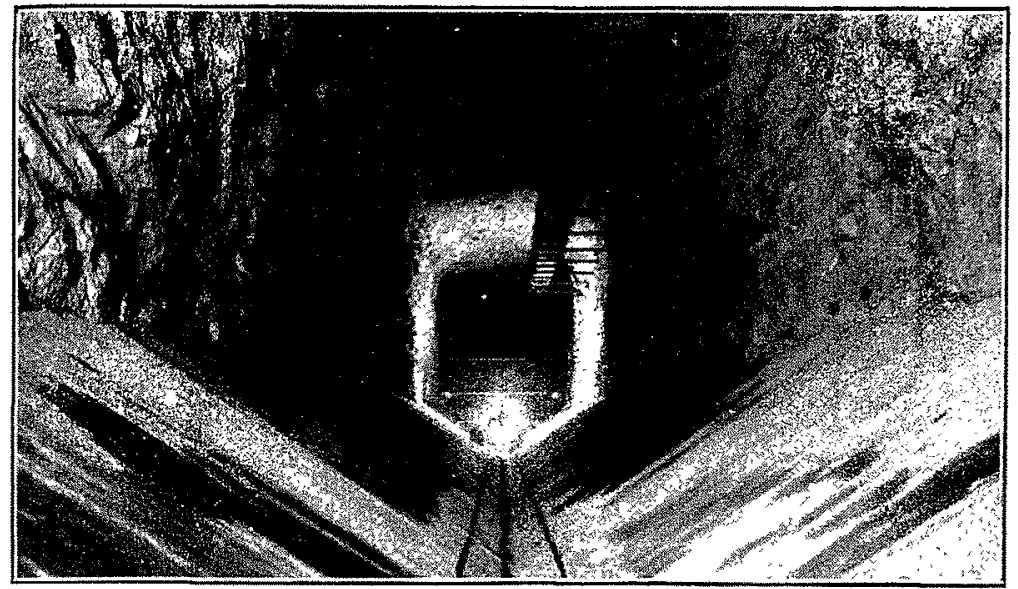

Fig. 3. - Dessableur de Tel (sur l'Adige) $35 \mathrm{~m}^{3} / \mathrm{sec}$.

Intćricur d'un bassin vu de l'aval, avec le purgeur, les fonds inclinés, la passerelle de service et, tout au fond, le tranquilliseur.

le limon non éliminé par lui et restant dans l'eau dessablée = cau motrice des turbines est, comme le fait voir le tableau 1 , extrêmement fin. Les $81,55+15,16=96,71 \%$ de son volume se composent de grains $<0,2 \mathrm{~mm}$.

Relevons encore que pendant les épreuves, la teneur en limon de l'eau dessablée a été de $119,95: 1.500=0,08 \mathrm{~cm}^{3}$ par litre. Le débit du dessableur étant d'environ $37 \mathrm{~m}^{3} / \mathrm{sec}$., la quantité de limon entraûnée vers les turbines en $24 \mathrm{~h}$., était ce jour-là de :

$$
\frac{0,08 \times 37 \times 1.000 \times 86.400}{1.000 \times 1.000}=254 \mathrm{~m}^{3}
$$

dont les $96,71 \%$, soit plus de $245 \mathrm{~m}^{3}$, étaient composés de grains $<0,2 \mathrm{~mm}$.

Pendant les mêmes épreuves, la leneur en alluvions de l'eau de purge prélevée a été de $2.518: 1.500=1,68 \mathrm{~cm}^{3}$ par litre, soit $1,68: 0,08=21$ fois plus forte que la teneur en limon de l'eau dessablée. Le prélèvement de l'eau de purge au moyen d'un seul tuyau n'ayant eu lieu qu'en un seul point sur le milieu du radier de la galerie d'évacuation, la teneur en alluvions de l'eau prélevée était certainement plus élevée que la teneur moyenne en alluvions de l'eau de purge. Ces alluvions prélevées de l'eau de purge peuvent servir pour une comparaison qualitative avec les alluvions (limon) de l'eau dessablée, mais la teneur qui en résulte pour l'eau de purge ne peut être utilisée pour calculer la quantité d'alluvions évacuées par le dessableur.

On peut se demander si, à Tel, l'efficacité du dessableur qui, du débit très important de $37 \mathrm{~m}^{3} / \mathrm{ser}$., permet d'éliminer la presque totalité des alluvions dépassant $0,2 \mathrm{~mm}$. n'a pas été poussée trop loin el si un ouvrage de dimensions moindres et moins coûteux, n'aurait pas été suffisant pour la prolection des tunnels, des canaux et aussi des turbines. L'exposé qui va suivre des résultats pratiques obtenus orientera le lecteur sur ce póint.

\section{EXPLOITATION DES TUNNELS ET DES CANAUX}

La courte période d'exploitation depuis la mise en service du dessableur, au printemps 1931, ne permet pas r'observer une nouvelle progression de l'usure des radiers des tunnels et des canaux; de plus, selon les cxpériences faites dans d'autres usines, on peut etre certain que le limon, dont Ia composition granulomérique donnée ci-dessus est extrêmement fine et qui est en suspension dans l'eau, ne pourra avoir qu'une action des plus minimes sur les enduits en ciment des radiers.

\section{EXPLOITATION DE L'USINE DE TEL}

Celle usine, construite il $\mathrm{y}$ a près de 30 ans par les "Etschwerke "= Usines de l'Adige, pour un débit de $15 \mathrm{~m}^{3} / \mathrm{sec}$, a été agrandie lors de la construction de celle de Marlengo ; elle est aujourd'hui propriété des "Azienda Elettrica Consorziale ", mais exploitée temporairement en partie par elles et en partie par la "Società Alto Adigen.

Avec le bassin de décantation primitif, la chambre de nise en charge, toujours en partie colmatée, remplissait mal son but de réservoir et de distributeur d'eau dans les deux conduites forcées ; certaines lurbines s'usaient rapidement.

Depuis la mise en service du dessableur, la chambre de. mise en charge est toujours entièrement libre de dépôts et les turbines s'usent beaucoup moins. Vu la courte périods: d'exploitation de deux ans et les conditions d'exploitation un peu compliquées de l'Lsine de Tel, les avantages que le dessableur a eu pour elle ne peuvent pas encore être donnés par des chiffres.

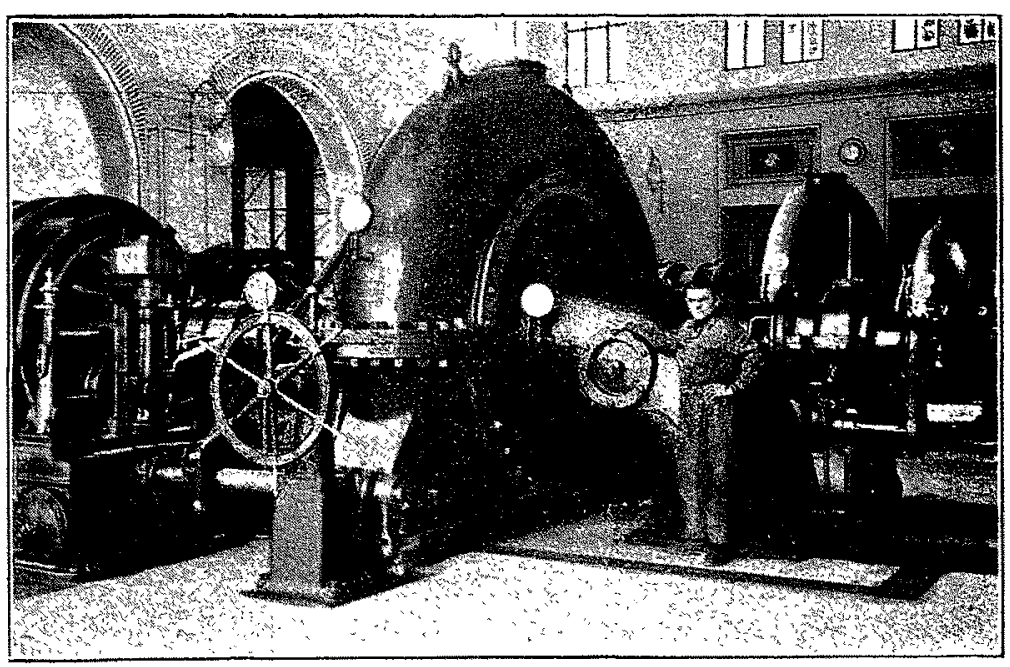

Fig. 4. - Turbine de l'Usine de Marlengo.

Dates de construction : $Q=7.500 \mathrm{l} / \mathrm{sec} . \mathrm{H}=128 \mathrm{~m}$., $\mathrm{n}=420 \mathrm{a} 500 \mathrm{p.m} . \mathrm{Ne}=10.300 \mathrm{ch}$.

\section{EXPLOITATION DES TURBINES}

\section{DE L'USINE DE MARLENGO}

Cette usine est équipée de cinq turbines Francis de $10.300 \mathrm{ch}$. chacune, dont quatre actionnent des génératrices ì courant continu. I.a fig. 4 montre une de ces turbines, la fig. 5 deux coupes de son distributeur et de sa roue. 
Avec le bassin de décantation primilif de la prise d'can, l'usure des turbines par les alluvions non éliminées telles que : graviers, sables et limons, se concentrait principalement sur les parois $P_{-} P$ et les aubes mobiles $A-A$ du distributeur, sur la roue $R$, sur les anneaux latéraux $J-J$ et sur le couvercle C. Au début de l'exploitation de l'usine, cette usurc, très rapide, a donné lieu à différentes avaries répa-

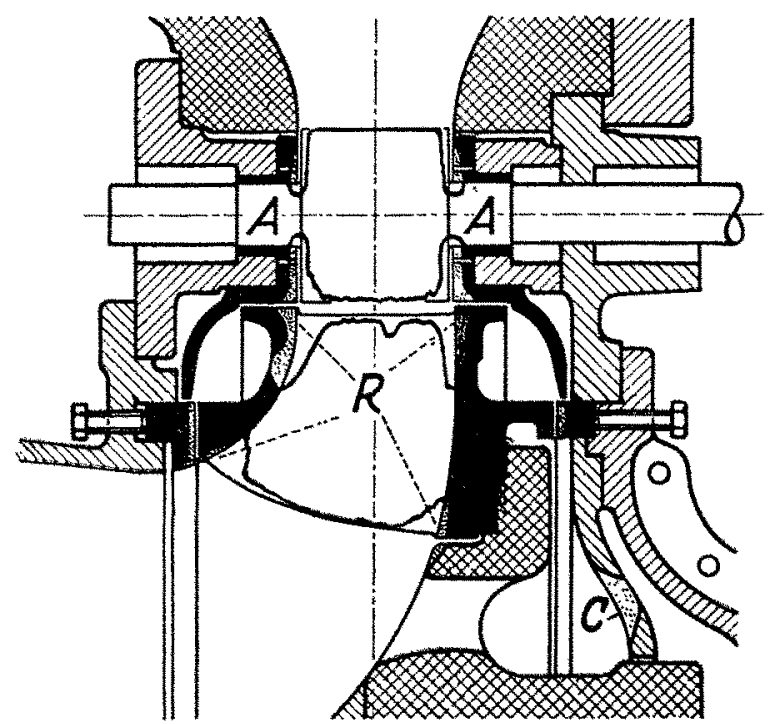

Tig. 5. - Coupes du distributeur et de la roue d'une turbine de Marlengo avec indieation de l'usure.

rables, mais qui, parfois, ont nécessité l'arrêt immédiat de la lurbine pendant un certain temps. La fig. 6 représente deux aubes directrices en acier coulé, après trois ans, la fig. 7 une roue en bronze spécial, après deux ans de service. (Nous regrettons de re pas posséder de photographies des parois $P-P$ usées). Sur la fig. 6, on remarquera la très forte usure de la pointe intérieure $p-p$ des aubes, diminuée de longueur et d'épaisseur jusqu'à former un véritable tranchant, en $t-t$ les profondes coupures de leurs tourillons.

La fig. 7 montre en $a, b, c$ et $d$ les ravages du sable sur les couronnes et les aubes de la roue. L'extrême usure des organes intérieurs de la turbine a été représentéc aussi bien que possible dans les coupes de la fig. 5

Après quelques années d'exploitalion avec le bassin primitif, le service de l'usine avait dû reconnaître que la durée des principales pièces des turbines soumises à l'usure, soit : les parois et les aubes du distributeur, la roue, les deux anneaux latéraux et le couvercle, ne pouvait dépasser deux ans. Chaque hiver, dès que le débit de l'Adige permetlait cette opération sans perte d'eau, il fallait done démonter les dites pièces usées et les remplacer par des nouvelles dans deux des quatre turbines actionnant les dynamos à courant continu. l'achat des nouvelles pièces ef leur ajustage dans les turbines représentaient un travail de longue haleine et excessivement coûteux. Si nous sommes bien rcnseignés, il est arrivé que le prix de la remise ì l'état neuf d'une seule turbine ait atteint la somme de 200.000 lires.

Depuis lat mise en service du dessablenr, l'uan ne conlient plus que du limon très fin dont nous avous donnć plus haul la composition granulométrique ; l'usure des lurbines a diminué dans une très forte proportion ef l'exploitation de l'usine a été grandement amélioréc. Pondant la première période d'été, en 1931, les quatre turbines pour le courant continu ont été en service ininterrompu saus la moindre

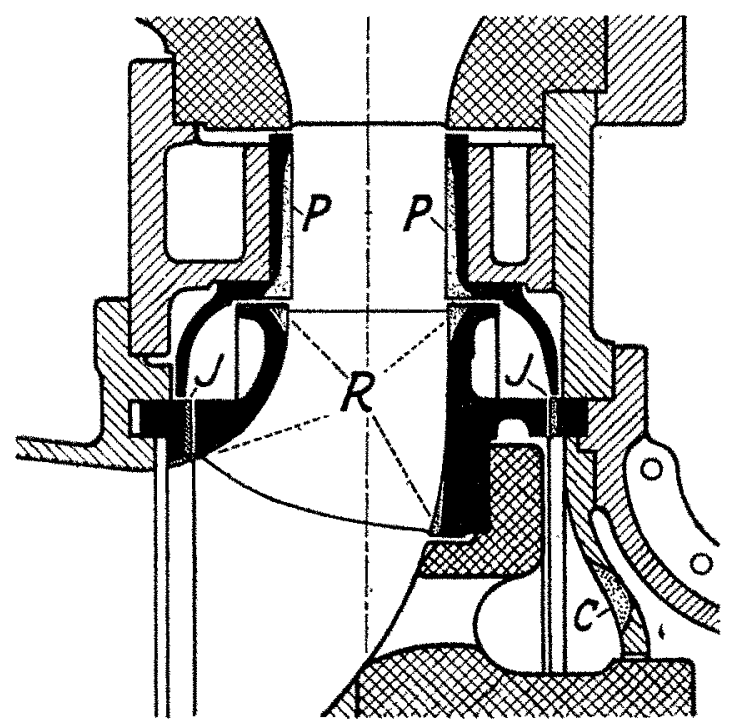

avarie. En février 1932, une des deux turbines remises à neuf en février 1931 fut ouverte pour un contrôle qui en révéla l'usure excessivement faible, presque imperceptible, comme le montre la fig. 8. La turbine put donc sans autre être referméc et remise en service. Seul le moyeu de la roue, en service depuis plusieurs années avec le bassin primitif, laisse voir une usure prononcée subie avant la mise en service da dessableur.

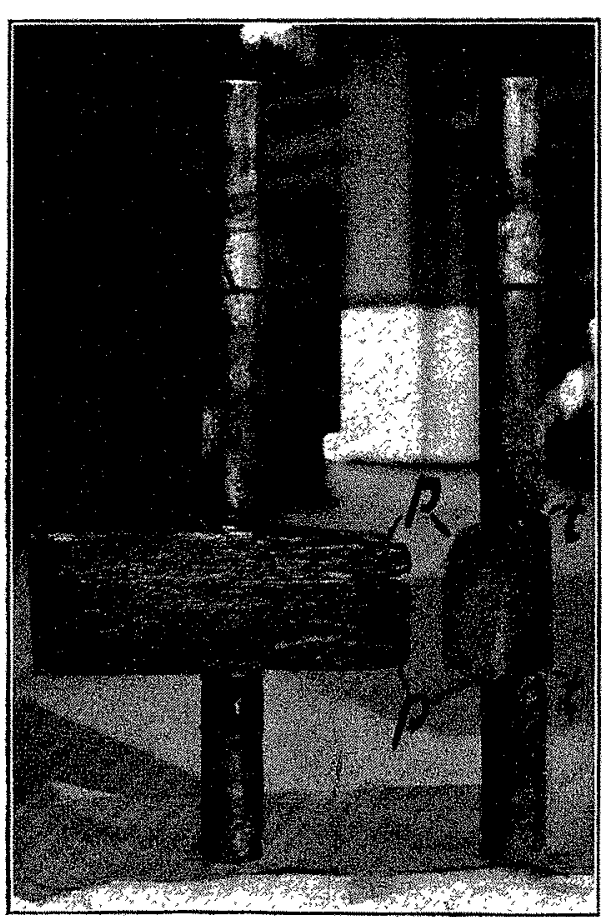

Fig. 6. - Aubes directrices d'une turbine de Marlengo après 3 ans de service avec le bassin de décantation primitif. A remarquer la très forte usure de la pointe $p \sim p$ et les profondes coupures $t-t$ des tourillons.
En février 1933, ce fut le tour de la seconde des denx turbines remises à neuf en février 1931 et ayant ainsi deux ans de service avec le dessableur, d'elr" ouverte. Les parois $P-P$ de son distributeur fig. 5 ; en acier, de $20 \mathrm{~mm}$. d'épaisseur, qui, autrefois, en deux ans, fortement usées et perforées en de nombreux endroits, étaient devenues absolument inutilisables, élaient en bon élat. La fig. 9 représente une de ces parois après deux ans de service avec le dessableur. Sur sa face on distingue faiblement la position des aubes et, entre celles-ci, le chemin tracé par l'eau limoneuse. La profondeur de ce chemin varie entre 0,6 et $1,5 \mathrm{~mm}$.Par place, aux extrémités des boîtes des aubes, la face de la paroi porte d'étroits sillons de 4 à $6 \mathrm{~mm}$. de profondeur. Sur la partie intérieure $r-r$ de la paroi, en retrait de la face, on voit encore toutes les traces du tour, l'usure y est donc nulle. Le service de l'usin:e fermera à la soudure les sillons aninur des boîtes el laissera les deux parois en service.

La fig. 10 montre deux aubes directrices en acier coulé de la même tur- 


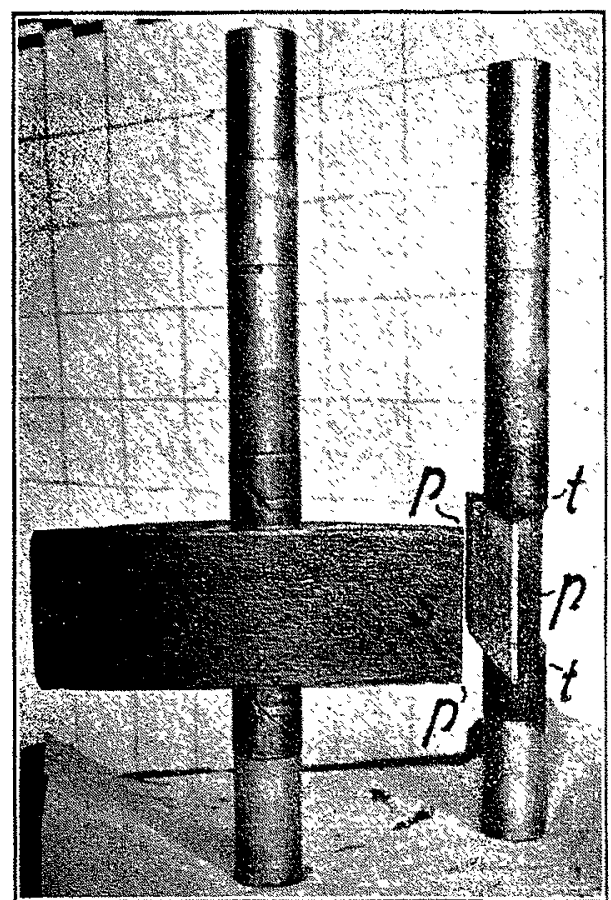

Fig. 10. - Aubes directrices d'une turbine de Marlengo après 2 ans de service avec le dessableur. La pointe de l'aube $p-p$ parầt intacte, en $t$ - $t$ les tourillons n'ont pas de coupures.

Turbines $\left\{\begin{array}{llllll}\text { Admissions : } & 100 \% & 75 & \% & 50 \% \\ \text { Rendements: } & 82,5 \% & 83,5 \% & 76,5 \%\end{array}\right.$
Dynamos $\left\{\begin{array}{lrllll}\text { Puissance : } & 100 & \% & 75 & \% & 50 \% \\ \text { Rendements : } & 95 & \% & 94 & \% & 92,5 \%\end{array}\right.$

Les mesures faites par le service de l'usine avant la construction du dessableur avaient montré que la diminution du rendement des turbines causée par leur très forte usure iétait, après deux ans de service, de $10 \%$ de leur rendement à l'état néuf. Nous avons admis que celte diminution de $16 \%$ avaït été constatée pour une admission moyenne de $75 \%$ et qu'après un an de service, toujours sans le dessableur, la diminution de rendement des lurbines était de l'ordre de $6 \%$.

En février 1932, avec le service -de l'usine, nous avons estimé que le rendement d'une turbine qui, depuis sa réparation, avait marché une année avec le dessableur et dont l'excellent état est visible sur la photographie fig. 8, deviait à peine avoir diminué. Comme chiffre de celte diminution de rendement nous avons néanmoins, pour nos calculs, admis lle chiffre rond de $1 \%$ certainement supérieur à la valeur réelle.

En février 1933, avec le même service, nous avons estimé que le rendement d'une turbine qui, depuis sa réparation, avait marché deux ans avec le dessableur et dont les pièces sont visibles sur les photographies fig. 9,10 et 11, pouvait avoir diminué de $3 \%$ au maximum.

Sur la base de ces chiffres de rendements et à l'aide des diagrammes de rendements d'une turbine Francis, de caractéristiques semblables à celles des lurbines de Marlengo, déterminés très cxactement, à l'état nouf el à l'état usé et représentés sur la fig. 12. nous avons, par analogie, établi pour la fig. 13 les courbes des rendements approximatifs probables des turbines de Marlengo : ì l'état neuf, après un

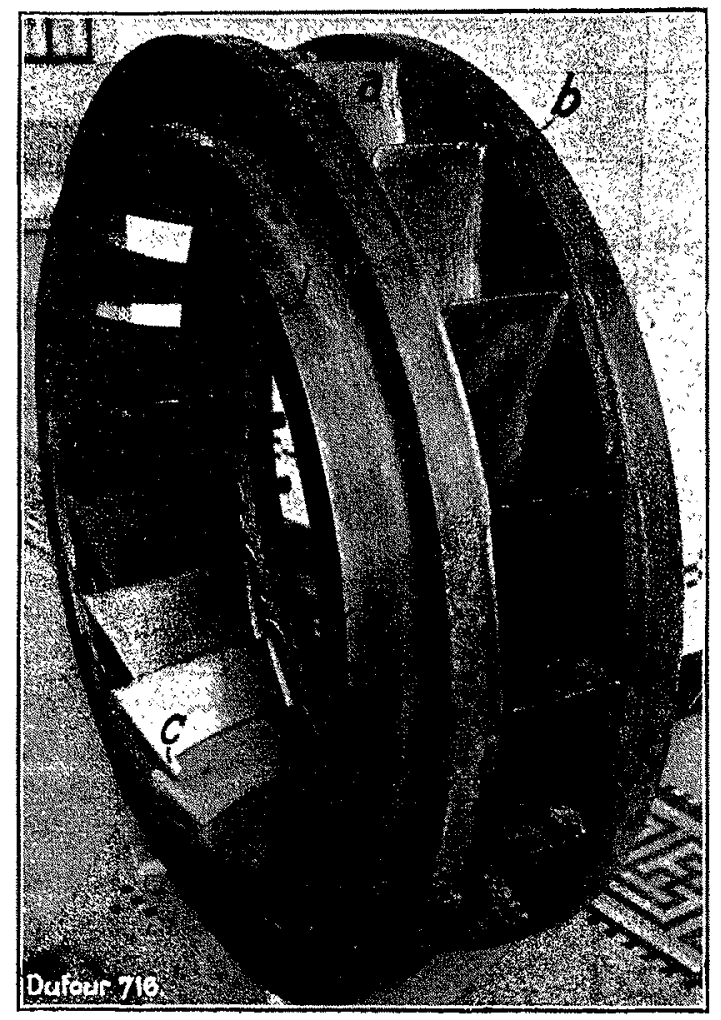

Fic. 11. - Roue en bronze spécial d'une turbine de Marlengo après 2 ans de service avec le dessablear.

été et après deux étés de service avec le bassin, après un étć et après deux étés de service avec le dessableur.

Avec les débits mensuels moyens, les chutes mensuelles moyennes, les rendements des dynamos cités, comme base, et, à l'aide dos courbes de la fig. 13. nous avons ensuite calculé, pour chaque mois de l'année 1932 par exemple, les puissances de l'usine en service dans les conditions de marche I, II et III précisécs à la page 5.

Voici quelques compléments d'explication sur ces calculs :

I. Puissance de l'usine avec des turbines à l'élat neuf. Pour ce calcul, les débits moyens mensuels disponibles en hiver et utilisables en été ont été, suivant leur importance, répartis par parts égales sur 2,3 ou 4 turbines de façon à obtenir pour celles-ci le meilleur rendement et pour l'usine la puissance la plus grande possible.

II. Puissance de l'usine en service avoc le bassin, c'est-àdire avec des turbines neuves et des turbines à différents degrés d'usure. Dans la répartition des débits disponibles en hiver el utilisables en été, par parts égales sur 2,3 ou 4 turbines, nous avons admis que les turbines les moins usées (celles n'ayant marché qu'un été ou celles remises à neuf) étaient en service, cela, toujours, pour obtenir les meilleurs rendements des turbines et la plus grande puissance possible de l'usine.

III. Puissance de l'usine en service avec le dessableur, c'est-à-dire avec des turbines neuves et des turbines à différenis degrés d'usure, celle usure étant toujours beaucoup plus faible que pour la condition de marche II. La répartition des débits disponibles en hiver et utilisables en été a été faite de la même façon que pour l'usine en service avec le bassin (condilion de marche II).

La manulention des turbines admise pour le calcul des séries de puissances I, II et III correspond à la manutention en vigueur à l'Usine de Marlengo avant la mise en service du 
Turbine Francis: $Q=4155 \mathrm{l} / \mathrm{s} . H=95,5 \mathrm{~m} . n=500 \mathrm{t} / \mathrm{min} . P=4350 \mathrm{ch}$.

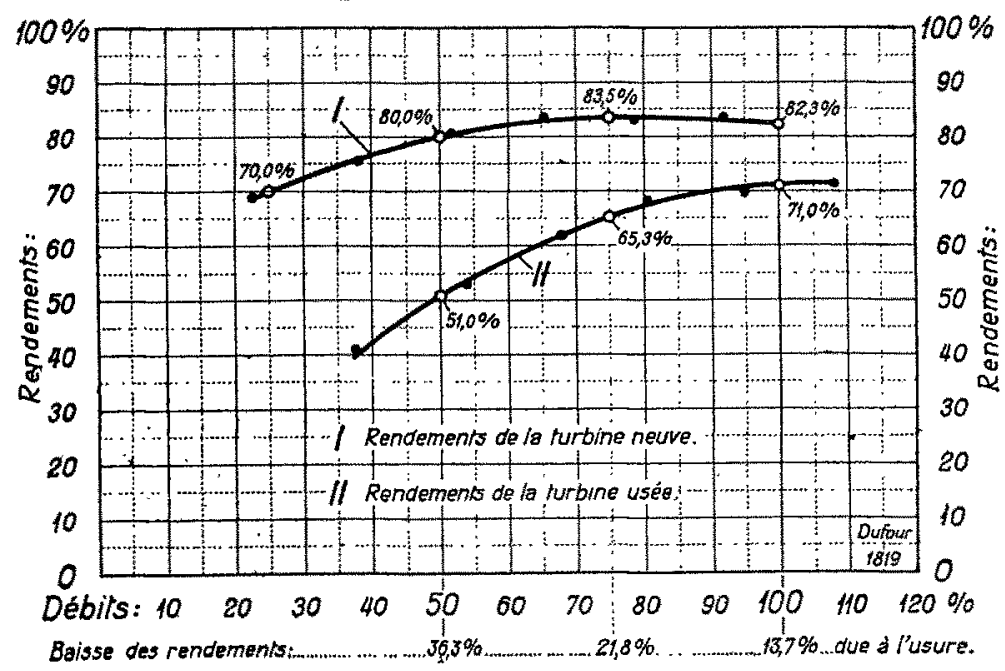

Fig. 12. - Rendements d'une turbine Francis aux caractéristiques semblables à celles de Marlengo et soumise à l'usure par les alluvions.

dessableur. Chaque année, en février et mars, pendant les plus basses eaux, les deux turbines les plus usées parce qu'en service depuis deux ans, étaient successivement arrêtées et remises à neuf. Dès le mois d'avril de chaque année, l'usine disposait donc de deux turbines en service depuis une année et en partie usées et de deux turbines à l'état neuf.

Les résultats des calculs que nous venons d'énumérer sont résumés sur la fig. 14 qui, pour chaque mois de l'année, donne : le débit et la chute mensuels moyens de l'usine, puis, pour ehacune des conditions de marche I, II et III, le rendement moyen des turbines et la puissance de l'usine.

Selon cette figure, la production d'énergie de l'Usine de Marlengo marchant avec de l'eau claire et des turbines neuves ne subissant pas d'usure serait de :

\section{$234070800 \mathrm{kWh}$.}

Pour l'usine marchant avec le bassin de décantation primitif, cette production, vu la forte usure des turbines, n'était plus que de :

$$
220841400 \mathrm{kWh} \text {. }
$$

et la perte d'énergic résultant de celte usure de :

$$
234070800-220841400=13229400 \mathrm{kWh} \text {. }
$$

soit le $5,66 \%$ de la production de l'usine avec des turbines neuves.

Pour l'usine marchant avec le dessableur, la production d'énergie, va la faible usure des turbines, était encore de :

$$
231577200 \mathrm{kWh} \text {. }
$$

et la perte d'énergie résultant de cette usure n'était plus que de:

$$
234 \quad 070800-231577200=2493600 \mathrm{kWh} \text {. }
$$

soit le $1,06 \%$ de la production de l'usine avec des turbines neuves.

La construction du dessableur a donc permis de récupérer

$13229400-2493600=10735800 \mathrm{kWh}$. soit le 4,6\% de la production de l'usine avec des turbines neuves et le $81 \%$ de la perte d'énergie subie par l'usine pendant sa marche avec le bassin de décantation primitif.

Avec le bassin primitif, l'usure rapide des turbines rendait parfois inévitable leur arrêt pour des réparations urgentes et, ces arrêts d'un groupe générateur se produisant presque toujours en temps de crue, il en résultait pour l'usine une perte d'énergic appréciable et nuisible à la marche régulière des fabrications. Avec le dessableur et l'usure très lente des turbines aucun arrêt pour réparation urgerite n'a été nécessaire.

Dans nos calculs, nous avons admis que le débit de l'usine était toujours réparti sur les turbines les moins usées. Ce sont là des conditions de marche idéales qui ne sont pas réalisées. car parfois, pour reviser un groupe dont la turbine est neuve, il est nécessaire d'avoir recours au groupe voisin dont la furbine est usée. Comme dans les conditions de marche III, la diminution de puissance due à cette usure est bealucoup plus faible que dans les conditions II, il en résulte que la production d'énergie de l'usine est bien supérieurc.

Dans ces deux cas nous sommes en présence d'une récupération d'énergie, due au dessableur, qui n'a pas pu ètre chiffrée et n'est pas comprise dans les chiffres ci-dessus.

\section{RESLME ET CONCLUSIONS}

L'exposé qui précède montre qu'à l'Usine de Marlengo dont l'eau dérivée de l'Adige, gros torrent de montagne, charrie des graviers, des sables et des limons, l'épuration de l'eau au moyen d'un grand bassin de décantation était insuffisante. Les alluvions qui, malgré ce bassin, traversaient encore les turbines, usaient leurs distributeurs et leurs roues motrices en deux ans et causaient à l'usine des arrêts et des pertes d'énergie importantes.

Le Dessableur Dufour mis en service au début de 1931 élimine d'une façon continue et sûre non seulement toutes

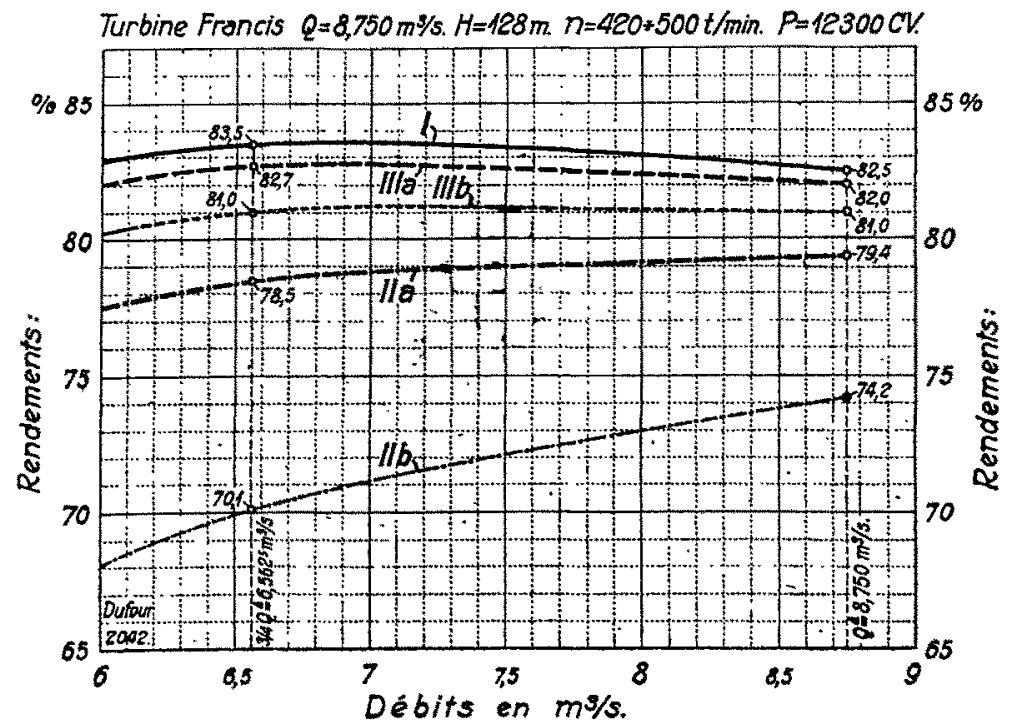

Fig. 13. - Rendements des turbines de Marlengo : I. avec des turbines neuves

Avec le bassin primitif : IIa, après un été - IIb, après deux étés Avec lo dessableur : IIIa, après un été - IIIb, après deux étés 
les alluvions dépassanl $0,5 \mathrm{sinm}$, mais aussi une forte proportion d'éléments plus pelits. Le limon de l'eau dessablée no contient que $3,29 \%$ on volume de grains dépassant $0,2 \mathrm{~mm}$.

Une expérience de deux ans a montré que cetle épuration très efficace de l'eau motrice permet de prolonger la durée
Un procédé sommaire pour délerminer approximativemont l'énergic récupérée par le dessablcur serait peul-être le suivant: Avanl la construclion du dessableur, el aujourd'hui encore, le scrvice de l'usine déterminait el délermine chaque jour le débit de l'usine pour obtenir, à la fin de chaque année, le débit annuel moyen utilisé. Nous présumons

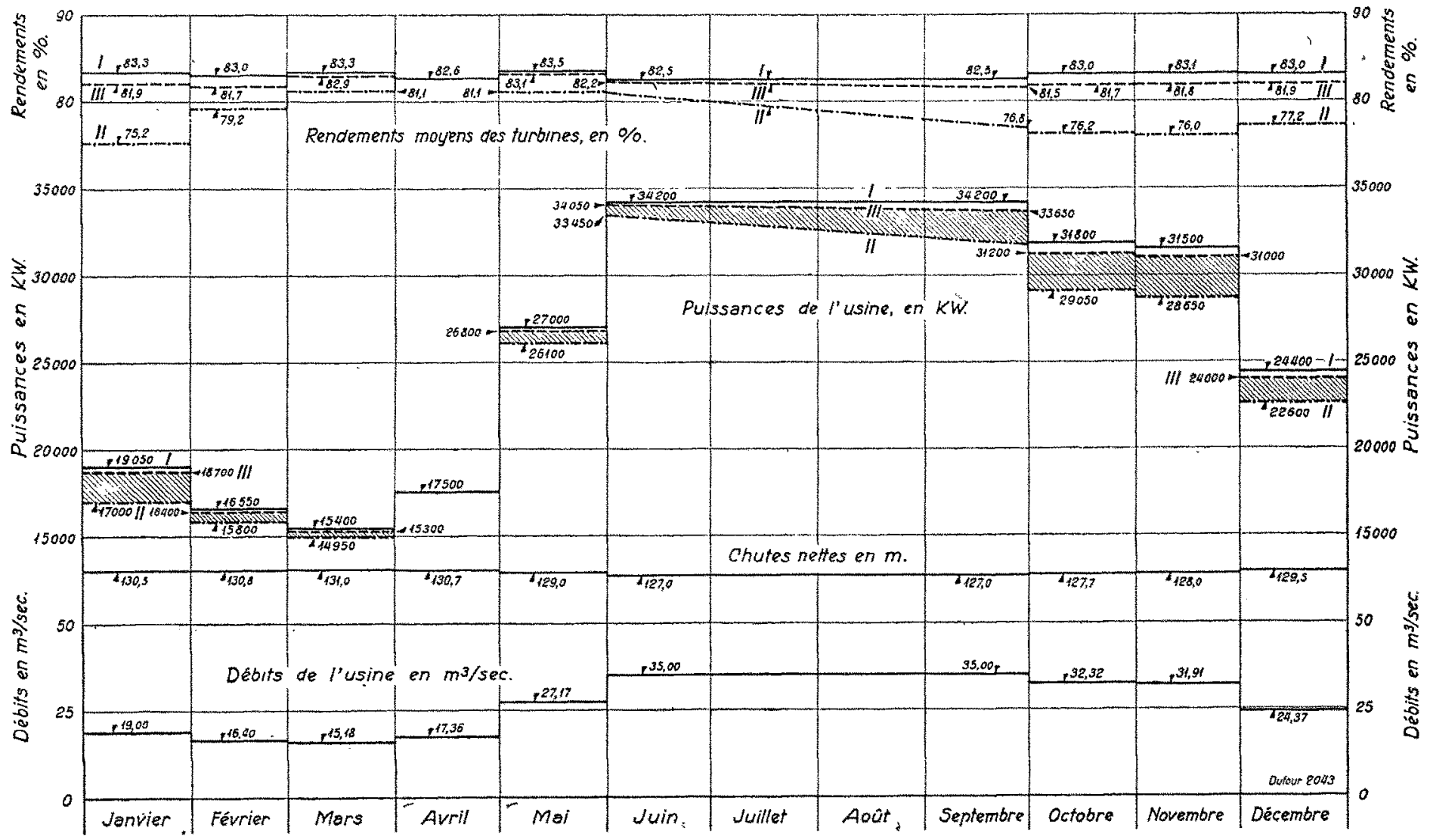

Fig. 14. - Débits, chutes, rendements moyens des turbines et puissances de l'Usine de Marlengo :
I. Aree des turbines neuves
II. Avec le bassin de décantation primitif
III. Avec le dessablemr

des distributeur's et des roues de turbines dans une forte proportion qui ne pourra être chiffrée exactement que dans plusieurs années.

Pendant la marche de l'usine avec le bassin de décantation primitif, la perte d'énergie due aux mauvais rendements des turbines usées était de l'ordre de $5,66 \%$ de l'énergie disponible avec des turbines neuves.

Depuis la mise en service du dessableur, cette perte d'énergie de l'usine n'alteint plus qu'environ le 1,06\%. La récupération par le dessableur se monte donc à environ $81 \%$ de la perte d'énergie subie avec le bassin primitif.

Dans quelques années, les stalistiques de l'Usine de Marlengo permetlront de délerminer l'économie réalisée, grâce au dessableur, sur le cô̂t de l'entretien des turbines.

La détermination exacte du gain d'énergie sera plus difícile, car elle implique la connaissance du rendement des lurbines neuves el des turbines à différents degrés d'usure, avec le bassin primitif et avec le dessableur, et, pour cela, le jaugeage exact du débil de ces machines prises isolément ou par groupes de deux au maximum. C'est pour celte raison que nos calculs ont dû être basés sur une mesure globale du service de l'usine et sur notre estimation guidée elle-mêmo par de nombreuses mesures de rendemonts sur d'autres turbines neuves et à différents degrés d'usure. qu'il établit aussi la production annuelle de l'usine en kilowatt-heures et pourrait, aujourd'hui encore, déterminer lo nombre de kilowatt-heures produit par $\mathrm{i} \mathrm{m}^{3} / \mathrm{sec}$. "de débit, en une année, avant et après la mise en service du dessableur.

\section{Le rapport :}

Nonibre de $\mathrm{kWh}$. produit par $1 \mathrm{~m}^{3} / \mathrm{sec}$. avec le dessableur Nombre de $\mathrm{kWh}$. produit par $1 \mathrm{~m}^{3} / \mathrm{sec}$. avec le bassin

donnerait la proportion dans laquelle la production d'énergie de l'usine avec le bassin primitif a élé augmentée par la mise en service du dessableur. L'exactilude de cette proportion dépendrait de l'exactitude du jaugeage des débits et de celle des compteurs d'énergie.

Aujourd'hui déjà, il nous parâ̂t certain, et c'est aussi l'opinion du service de l'Usine de Marlengo, que l'efficacité très poussée du dessableur de Tel n'est nullement exagéréc. Nous nous empressons toutefois dajouter que cette conolusion ne peut pas être généralisće à d'antres usines dont les transports d'alluvions et les conditions d'établissement seraient différents de ccux de Marlengo.

Par l'exposé des résultats obtenus à l'Usine de Marlengo, nous avons cherché, une fois de pỉus, à montrer l'importance que peut avoir pour une usine hydro-électrique dont les lurbines sonffrent de l'usure, l'installation d'un dessableur vëritablement officace. 